\title{
Oportunidades de integración de la realidad virtual al proceso de enseñanza de los estudiantes de ingeniería eléctrica: un análisis desde el enfoque de sistemas
}

\author{
Jesús Alberto Flores Cruz, Instituto Politécnico Nacional, México \\ Patricia Camarena Gallardo, Instituto Politécnico Nacional, México \\ Elvira Avalos Villarreal, Instituto Politécnico Nacional, México
}

\begin{abstract}
Resumen: La formación de ingenieros en cualquier rama del conocimiento ha representado a lo largo del tiempo un gran reto para las Instituciones de Educación Superior. Las exigencias de los sectores productivos para los profesionistas recién egresados en materia de conocimientos, habilidades y destrezas, resultan cada vez mayores. Esto ha motivado a algunas escuelas a adoptar estrategias que permitan que sus egresados afronten con éxito dichos requerimientos. Dentro de estas estrategias el uso de la Tecnologías de Información y Comunicación en el procesos de enseñanza-aprendizaje se ha destacado, por el papel que dichas tecnologías juegan en las tareas de búsqueda, recolección y utilización de la información, hasta llegar a los usos más recientes, donde a través de nuevas aplicaciones, se trasmiten conocimientos a los estudiantes de una forma muy parecida a la que les brindan las actividades de la ingeniería reales. Y es ahi que esta investigación tiene su sustento, analizando las posibles aplicaciones de la Realidad Virtual en la enseñanza de la ingeniería eléctrica, para complementar las practicas docentes tradicionales.
\end{abstract}

Palabras clave: realidad virtual, enseñanza de la ingeniería, ingeniería eléctrica

\begin{abstract}
The training of engineers in any branch of knowledge has represented over time a great challenge for higher education institutions. The demands of productive sectors for recent graduates in knowledge, skills and abilities, are increasing. This has prompted some schools to adopt strategies that allow their graduates successfully face up to these requirements. This has prompted some schools to adopt strategies that allow their graduates successfully face up to these requirements. One such strategy is the use of Information and Communication Technologies in the teaching-learning process has been highlighted by the role that these technologies play in the search effort, collection and utilization of information. This research has its basis, analyzing the potential applications of virtual reality in teaching electrical engineering, to complement traditional teaching practices.
\end{abstract}

Keywords: Virtual Reality, Engineering Education, Electrical Engineering

\section{Introducción}

ctualmente existe en México y en algunos países de Latinoamérica una pérdida de competi$\triangle$ tividad derivada de factores como la falta de inversión, la formación de talentos y la pérdida 1 de sentido de las políticas de desarrollo del país, sin que al parecer las entidades involucradas se hayan dado cuenta de las implicaciones que esta situación tiene para el desarrollo nacional, pues ante esta carencia es muy difícil competir en un mundo cada vez más globalizado (Academia de Ingeniería, 2010). Ulloa (2008) destaca al menos dos factores importantes que contribuyen con ese déficit, el primero de ellos está basado en el hecho de que la ingeniería ha perdido la relevancia social que tuvo en otros tiempos, pese a que en la actualidad esta profesión sigue siendo el artífice de las grandes obras de infraestructura de cualquier país, estas ya no resultan tan sorprendentes para el común de la sociedad como lo fueron en otra época; y el segundo factor y sin duda uno de los más importantes, el hecho de que existen condiciones que inciden en una deficiente preparación de los estudiantes de ingeniería, y es que durante la formación de un ingeniero se requiere que éste

Revista Internacional de Aprendizaje en Ciencia, Matemáticas y Tecnología

Volumen 1, Número 2, 2014, <http://sobrelaeducacion.com>, ISSN 2386-8791

(C) Global Knowledge Academics. J.A. Flores Cruz, P. Camarena Gallardo, E. Avalos

Villarreal. Todos los derechos reservados. Permisos: soporte@gkacademics.com 
adquiera una preparación integral e interdisciplinaria en materia de ciencias y matemáticas, que le permita entender los problemas complejos de la ingeniería, la biología, el medio ambiente, la propagación de enfermedades y epidemias, entre otras cosas (Bosch et Al., 2011), aunado al hecho de que también es necesaria una gran formación práctica algunas veces en el laboratorio y otras en la industria (Maynard et al., 2012), esta ultimas se ve restringida muchas veces por diversos factores entre los que se incluyen las posibles demandas a las que se enfrentaría una empresa si un estudiante se accidentara dentro de sus instalaciones durante una visita industrial, o por los costos y la logística necesaria para que esta actividad se lleve a cabo, lo cual frustra en gran medida los esfuerzos que hacen los administradores de la planta y el personal de la universidad para llevar a cabo esta acción.

Otra dificultad que incide directamente sobre la formación práctica de los estudiantes a través del contacto con las obras de ingeniería reales tales como la construcción de una estructura, un sistema, una red o un proceso, es el hecho de estas se llevan a cabo en plazos muy largos de tiempo, lo que representa, que los estudiantes suelan ver en una visita industrial sólo una pequeña fracción de todo el ciclo de vida del proyecto, perdiendo con ello la oportunidad de tener contacto por más tiempo con el contexto real de los sistemas productivos, quedando su conocimiento restringido en el mejor de los casos a la teoría que obtuvo en el salón de clases y a unas cuantas imágenes que se formo de lo que es la realidad en las obras de ingeniería.

Otro autor que también hacia énfasis en las dificultades que se presentaban en la enseñanza de la ingeniería fue Astin (citado por Sulbaran y Baker, 2000) quien describía en su monumental estudio de casi 25,000 estudiantes en más de 300 escuelas, que sólo el $43 \%$ de los estudiantes de primer año de ingeniería se graduaba, y que la explicación de este fenómeno implicaba un complejo conjunto de factores, destacándose entre ellos principalmente la actitud del estudiante, y la actitud que éste tenía hacia la ingeniería, la cual estaba fuertemente relacionada con su entorno educativo, destacando que uno de los factores que más llamaba la atención era el hecho de que aunque la mayoría de los estudiantes eran aprendices visuales, los entornos educativos a lo largo de la historia han estado llenos de prácticas verbales y que en la mayoría de las clases de la universidad se presentaba muy poca información visual, los estudiantes escuchan en todos ellas las conferencias que dictaban sus profesores y leían el material que estos escribían en el pizarrón o el que estaba escrito en sus libros de texto y en múltiples documentos dándole prioridad al componente de aprendizaje memorístico. Pero desafortunadamente, al ser la mayoría de los estudiantes aprendices visuales, estos no obtienen la información suficiente que a cambio les darían las presentaciones visuales dentro de la clase.

Lo anterior ha representado históricamente un gran reto para todas las instituciones de educación superior que participan en la formación de ingenieros, obligándolas continuamente a innovar la práctica docente, buscando estrategias que les permitan involucrar a sus estudiantes de manera efectiva en todas las etapas del ciclo de vida de un proyecto desde su concepción hasta su realización, por lo cual se presenta en esta investigación una posible solución ante estas necesidades tratando de conjuntar a la industria y los estudiantes dentro del salón de clases a través de los entornos virtuales de aprendizaje basados en Realidad Virtual (RV), o de la tecnologías relacionadas con la misma, ya que como argumenta textualmente Sampaio (2000), "los métodos tradicionales de educación responden solamente a las necesidades de algunos estudiantes en algunos temas específicos, pero en la mayoría de los caso no ofrecen un aprendizaje óptimo", indicando que la RV ofrece en cambio la oportunidad de proporcionar educación a los ingenieros de una forma que no es posible hacerlo con los métodos tradicionales, ampliando así la gama de herramientas disponibles para los profesores, con el fin de lograr llegar a más estudiantes y más asignaturas. Para utilizar esta nueva herramienta de manera óptima y eficaz, es necesario no sólo producir y entregar simulaciones educativas basadas en RV, sino también estudiar los diferentes mecanismos por los cuales la información técnica puede ser entregada a través de la RV y de la efectividad relativa de los diferentes desarrollos y plataformas de aplicación.

En esta investigación se muestran los resultados de un análisis efectuado desde el punto de vista sistémico, el cual destaca las posibilidades que tiene la RV para ser aplicada de manera generalizada dentro del proceso formativo de los estudiantes de ingeniería eléctrica, principalmente en aquellas asignaturas que representan alguna dificultad para los métodos de enseñanza tradicionales, entre los 
cuales se incluyen los conceptos complejos o abstractos, las situaciones riesgosas, los modelos complicados o alguna otra características que dificulte su comprensión por parte de los estudiantes (Bohorquez, et al., 2009).

\section{Contexto}

El contexto en el que se desenvuelve esta investigación se encuentra definido por el proceso de integración de la RV al proceso de formación de los estudiantes de ingeniería eléctrica, entendiéndose esta como aquella que tiene por objetivo: “...planear, proyectar, diseñar, innovar, controlar, instalar, construir, coordinar, dirigir, mantener y administrar equipos y sistemas, aparatos y dispositivos, destinados a la generación, transformación y aprovechamiento de la energía eléctrica en todas sus aplicaciones, asi como operar equipos y materiales eléctricos tomando en cuenta su interrelación con los sistemas de potencia, distribución y utilización; además de participar en la construcción, mantenimiento, conservación y administración de la planta productiva, con una visión integral del desarrollo social, económico e industrial del país." (ESIMEZ-IPN, 2013), ofertada por la Escuela Superior de Ingeniería Mecánica y Eléctrica, institución pública mexicana perteneciente al Instituto Politécnico Nacional. Y es en ese contexto educativo donde se analizan las oportunidades para la incorporación de la RV como una estrategia didáctica, principalmente para la trasmisión de conocimientos en aquellas áreas donde los métodos tradicionales no han logrado hacerlo con el éxito esperado, como es la enseñanza de conceptos científicos complejos o riesgosos (Nadan et al., 2011). Partiendo de la factibilidad que da la convergencia tecnológica en materia de hardware y software, la cual ha permitido el uso de esta tecnología en más universidades por la accesibilidad de costos (Abulrub, Attridge \& Williams, 2011) y la capacidad de trasmitir información de manera multisensorial, que hace que el usuario tenga vivencias que lo lleven a situaciones muy parecidas a las que enfrenta en la realidad. Lo anterior hace que la RV sea una herramienta con gran potencial cuando se requiere trasmitir al estudiante conceptos complejos y de situaciones en las que se deben extremar precauciones por el riesgo implícito que ellas tienen, como el manejo de sustancias químicas, altas temperaturas, visitas a plantas industriales, variables eléctricas elevadas, entre otras, ya que durante su formación profesional, en la mayoría de las veces, solamente se les muestran con el único propósito de conocer los elementos que los integran, sin tener la posibilidad de manipularlos u operarlos.

\section{Marco de referencia}

El marco de referencia en el que se encuadra esta investigación está definido por tres ejes principales, el enfoque de sistemas, la convergencia tecnológica y la realidad virtual.

Desde el enfoque de sistemas toda la sociedad está organizada mediante sistemas complejos, de esta forma la humanidad ha tratado de darle una apariencia de orden a su universo. Algunos sistemas como los informáticos son manejables y poco complejos, pero por otra parte, sistemas como los económicos, educativos, sociales o culturales, son sistemas muy grandes y complejos, haciendo que los métodos tradicionales de interpretación se vean limitados cuando se quieren estudiar dichos sistemas. En este estudio se utilizó la etapa de análisis del modelo de enfoque sistémico planteado por Clifton B. Chadwick (1992) como marco de referencia, en él se aplican los conceptos de enfoque y análisis de sistema en el ámbito educativo.

Por otra parte para Olawuyi y Mgbole (2012) "La convergencia tecnológica es la tendencia de los diferentes sistemas tecnológicos para evolucionar hacia la realización de tareas similares", en este caso de estudio esta evolución es hacia la RV. Mientras que Papadakis (2007) es más específico al mencionar que "la convergencia tecnológica es el proceso por el cual las telecomunicaciones, la informática y los medios de comunicación, sectores que en un principio operaron de forma independiente entre sí actualmente están creciendo juntos”. La convergencia tecnológica en las TIC está integrada por cinco grandes ejes, los cuales pueden presentarse en forma aislada o a través de combinaciones, pero siempre dependen del desarrollo tecnológico existente según Ojeda (citado por 
Yamakawa, et Al. 2010): la convergencia de redes, terminales, servicios, contenidos, y usos y aplicaciones. La convergencia de redes es una misma red que soporta distintos servicios y contenidos; por ejemplo, las redes de telecomunicación y teledifusión. La convergencia de terminales supone una misma terminal que da acceso a diferentes redes y servicios; por ejemplo, televisores, computadoras y teléfonos móviles. La convergencia de servicios supone un mismo servicio que se adapta a distintos contenidos; por ejemplo, televisión, video, telefonía fija-móvil e Internet. Por su parte, la convergencia de contenidos supone un mismo contenido adecuado para diferentes redes y servicios, como datos, audio e imágenes multimedia. Por último, la convergencia de usos y aplicaciones supone la misma red y la misma terminal que se utilizan para diferentes contenidos y servicios.

El tercer eje es el de la Realidad Virtual de la cual existen diversas definiciones interpretándola en algunos casos como una combinación de tres perspectivas (Brudniy \& Demilhanova, 2012), ver figura 1. Desde la perspectiva filosófica la RV se le considera como el área de interacción posible del sujeto, por otra parte desde la perspectiva técnica la RV tiene es considerada desde las tecnologías informáticas y las interacciones que con ella se producen y finalmente desde la perspectiva psicológica la RV se entiende como la modificación artificial que se hace de la realidad y de cómo los sujetos se comporta dentro de esta modificación.

Figura 1: El constructo de la Realidad Virtual

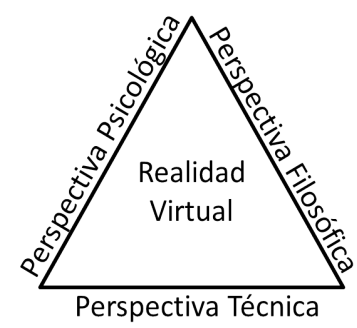

Fuente: Brudniy \& Demilhanova, 2012.

Para los fines de este estudio la RV se entiende como la forma más avanzada de relación entre una usuario y un sistema de información, dicha relación permite una interacción directa entre el usuario y el ambiente generado artificialmente, mismo que está destinado a estimular los sentidos humanos como son la vista, el oído, el tacto y más recientemente el olfato, caracterizándose principalmente por crear una ilusión de participación directa en el ambiente artificial. Islande (citado por Fällman, Backman, y Holmlund, 1999), plantea que existen términos como entorno sintético, ciberespacio, realidad artificial, tecnología de simulación, los cuales han sido adoptados como sinónimos de termino de RV por la gran cantidad de usos que ésta tecnología ha tenido, debido a que en la actualidad sus costo han bajado, a lo simple de su método, a la facilidad de uso y las ventajas de solucionar con facilidad los problemas que antes requerían complicados y costosos equipo especializado para mostrar los escenarios de la vida real.

\section{Desarrollo}

Al tratarse de un estudio analítico descriptivo, este se llevo a cabo mediante una revisión exhaustiva de diversas fuentes documentales indexadas principalmente en el Journal Citation Report ${ }^{\circledR}$, y de diversos artículos científicos relacionados con los temas involucrados; lo que sirvió como base para llevar a cabo esta investigación siguiendo una secuencia de análisis sistémico integrada por siete etapas, la cual se ilustra en la figura 2: 
Figura 2: Secuencia del análisis sistémico

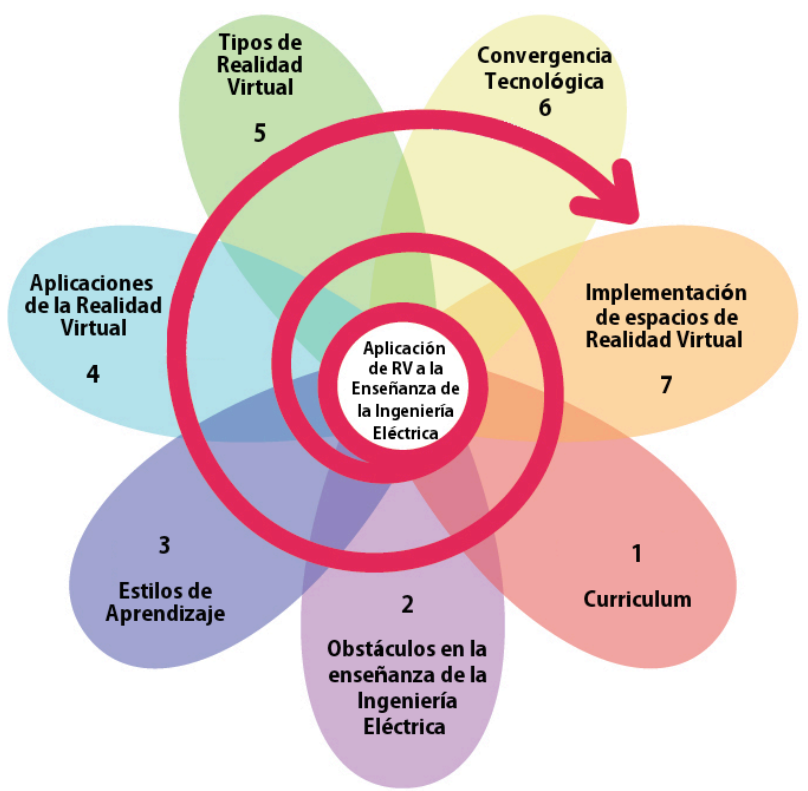

Fuente: Adaptación de Hope, 2011.

De la figura anterior se dio inicio a la secuencia sistémica con el análisis del plan de estudios de la carrera de ingeniería eléctrica, correspondiente a la etapa 1, con el fin de determinar aquellas asignaturas que representaban algún obstáculo dentro del sistema didáctico información que sirvió para generar la etapa 2 de la secuencia de la figura 2.

Para este fin fueron analizados los obstáculos didácticos definidos por Camarena (2009) como aquellos que son provocados por el profesor por el modo como este enseña los conocimientos de acuerdo a un modelo educativo específico, encontrándose que dichos errores pueden surgir fácilmente cuando una asignatura:

a) Incorpore conceptos abstractos o complejos que al tratar de ser enseñados a través de los métodos tradicionales representen alguna dificultad para el profesor, por ejemplo en el caso donde debe enseñar el concepto de átomo o enlace químico y para ello se auxilie de un dibujo en dos dimensiones hecho en el pizarrón, el cual por más detalles que contenga y por más esfuerzos que represente para el profesor, no ofrecen la misma cantidad de información que si éste utilizara un modelo 3D para trasmitir el conocimiento.

b) Requiera de ejemplificar maquinas o situaciones reales, por ejemplo un trasformador eléctrico dibujado en el pizarrón no ofrece la misma información que una fotografía tridimensional del mismo y menos aún que un modelo tridimensional presentado en un espacio de RV.

c) Genera situaciones de riesgo para los estudiantes, entendiéndose estas como aquellas donde exista peligro de lesión o muerte, ocasionado por causas relacionadas directa o indirectas con la electricidad. Entre los riesgos eléctricos más comunes a los que se enfrenta un estudiante son provocados por:

i) Las descargas eléctricas que puede recibir por el contacto directo o indirecto a través de algún equipo electrificado, onda de choque o arco eléctrico, cuando exista alguna falla.

ii) Una descarga eléctrica por diferencia de potencial.

iii) La inhalación de los gases tóxicos generados por la falla en un equipo eléctrico.

v) El fuego resultante de una falla eléctrica. 
Durante el desarrollo de alguna práctica o una visita industrial existen situaciones que de no ser manejadas con las medidas de seguridad necesarias pueden ocasionar un accidente, como ejemplo de lo anterior podemos citar la situación en la que se tiene que visitar una subestación eléctrica elevadora cuyos voltajes de operación están en el rango de los $13.8 \mathrm{kV}$ a los $500 \mathrm{kV}$.

d) Requiera de visitas industriales a lugares específicos del sector eléctrico, por ejemplo la visita a una planta hidroeléctrica o termoeléctrica con el fin de conocer cómo el proceso de generación de la electricidad.

e) Requiere de conocer la fabricación de algún equipo o material específico. En esta categoría caen las visitas industriales a las plantas de manufactura, por ejemplo las que se llevan a cabo para que los estudiantes conozcan los procesos de fabricación de los equipos eléctrico.

Las revisiones del plan de estudios y sus contenidos temáticos anteriores, permitió determinar y clasificar en que asignaturas existe alguna situación de las descritas en el listado anterior (ver tabla 1):

Tabla 1: Resultado del análisis sistémico aplicado al plan de estudios de Ingeniería Eléctrica

\begin{tabular}{|c|c|c|c|c|c|}
\hline \multirow[b]{2}{*}{ Asignatura } & \multicolumn{5}{|c|}{ Características de la asignatura } \\
\hline & $\mathbf{C A}$ & GSR & MRR & RVI & RCE \\
\hline Matemática & $\bullet$ & & $\bullet$ & & \\
\hline Química & $\bullet$ & $\bullet$ & $\bullet$ & & \\
\hline Física & $\bullet$ & & $\bullet$ & & \\
\hline Computación & $\bullet$ & & & & \\
\hline Humanidades & & & $\bullet$ & & \\
\hline Economía & & & $\bullet$ & & \\
\hline Administración & & & $\bullet$ & & \\
\hline Circuitos eléctricos & $\bullet$ & $\bullet$ & $\bullet$ & & $\bullet$ \\
\hline Electrónica & $\bullet$ & $\bullet$ & $\bullet$ & & $\bullet$ \\
\hline Estructura de los materiales & $\bullet$ & & $\bullet$ & & \\
\hline Conversión de la energía & $\bullet$ & $\bullet$ & $\bullet$ & $\bullet$ & $\bullet$ \\
\hline Instalaciones eléctricas & $\bullet$ & $\bullet$ & $\bullet$ & $\bullet$ & $\bullet$ \\
\hline Equipo eléctrico & $\bullet$ & $\bullet$ & $\bullet$ & $\bullet$ & $\bullet$ \\
\hline Fuentes de generación & $\bullet$ & $\bullet$ & $\bullet$ & $\bullet$ & $\bullet$ \\
\hline Líneas y redes de distribución & $\bullet$ & $\bullet$ & $\bullet$ & $\bullet$ & $\bullet$ \\
\hline Protecciones eléctricas & $\bullet$ & $\bullet$ & $\bullet$ & $\bullet$ & $\bullet$ \\
\hline Maquinas eléctricas & $\bullet$ & $\bullet$ & $\bullet$ & $\bullet$ & $\bullet$ \\
\hline Sistemas de tracción & $\bullet$ & $\bullet$ & $\bullet$ & $\bullet$ & $\bullet$ \\
\hline Sistemas de iluminación & $\bullet$ & $\bullet$ & $\bullet$ & $\bullet$ & $\bullet$ \\
\hline Altas tensiones & $\bullet$ & $\bullet$ & $\bullet$ & $\bullet$ & $\bullet$ \\
\hline
\end{tabular}

CA: Conceptos Abstractos, GSR: Genera Situaciones de Riesgo, MRR: Requiere Modelos para Representar la Realidad, RVI: Requiere Visitas Industriales \& RCE: Requiere Conocer Equipo en Campo.

Fuente: Elaboración propia, 2014.

Etapa 3. Una vez encontradas las características de cada asignatura que podrían representar un posible obstáculo didáctico dentro del proceso de enseñanza aprendizaje, el siguiente paso fue efectuar un análisis para determinar cómo la tecnología de RV era compatible con los estilos de aprendizaje de la mayoría de los estudiantes, para lo cual fue necesario establecer que sentidos humanos son estimulados por dicha tecnología, encontrándose que estos sistemas estimulan principalmente tres de los cinco sentidos humanos: la vista, el oído y el tacto, y más recientemente y con menor uso el olfato (Ariyakul, Aizawa \& Nakamoto, 2013). Lo anterior permitió la elaboración de la tabla 2 donde se observa como la RV satisface tres de los estilos de aprendizaje más comunes que tiene los estudiantes. 
Tabla 2: Relación de la RV con los estilos de aprendizaje

\begin{tabular}{|l|c|c|}
\hline \multirow{2}{*}{ Estilo de aprendizaje } & \multicolumn{2}{|c|}{ Realidad Virtual } \\
\cline { 2 - 3 } & Semi- inmersiva & Inmersiva \\
\hline Visual & $\mathrm{si}$ & $\mathrm{si}$ \\
\hline Auditivo & $\mathrm{si}$ & $\mathrm{si}$ \\
\hline Lecto-escritura & no & no \\
\hline Kinestésico & $\mathrm{si}$ & $\mathrm{si}$ \\
\hline
\end{tabular}

Fuente: Elaboración propia, 2014.

Etapa 4. En la siguiente etapa se continuó con el análisis sistémico de la información, encontrando que la RV es utilizada y aplicada desde sus orígenes y hasta la actualidad en diversos campos del conocimiento humano, principalmente en:

a) Aplicaciones militares (RTO/NATO, 2003):

- $\quad$ Simuladores de vuelo.

- Simuladores de campos de batalla.

- Entrenamiento médico.

- Simulación de Vehículos.

- Campo virtual de entrenamiento.

b) Aplicaciones médicas (Kalorama Healthcare reports, 2011):

- Simulación de cirugías.

- Tratamiento de fobias.

- Cirugía asistida por robots.

- $\quad$ Formación profesional.

c) Aplicaciones de visualización científica (Leng, 2001):

- Procesamiento de datos a gran escala.

- Visualización a través de foto realismo o de videos 3D.

- Interfaces hombres-computadora.

- Expresión mediante modelos de conceptos científicos.

d) Aplicaciones en la ingeniería (Talaba y Amditis, 2008):

- Diseño de productos en 3D

- Modelado de productos en 3D

- Creación y prueba de prototipos virtuales en 3D

- Visualización en 3D

Lo anterior llevo a la elaboración de la figura 2 en la que se observan las cuatro categorías principales en las que se puede encuadrar el uso de la RV.

Figura 3: Principales usos de la RV

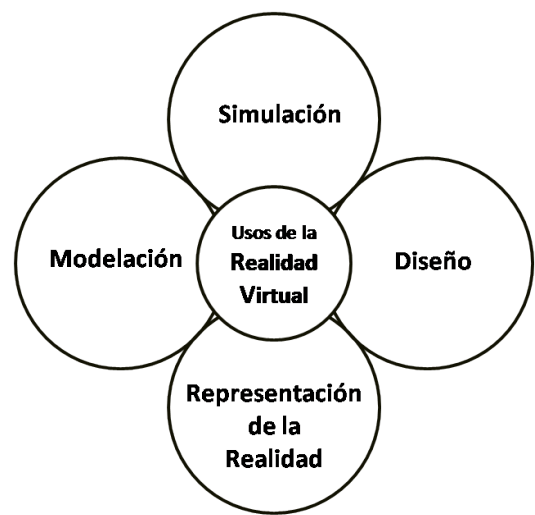

Fuente: Elaboración propia, 2014. 
En la Etapa 5 se abordaron los tipos de RV encontrándose que estos se pueden clasificar principalmente por los niveles de interacción y de inmersión que proporcionan al usuario, existiendo básicamente tres tipos (Fällman, Backman \& Holmlund, 1999; Ai-Lim y Wai, 2008):

a) El primero de ellos es la RV de escritorio, o RV no inmersiva que es la forma más común y menos costosa de RV que existe y que por lo general está conformada simplemente de una computadora de escritorio con características comunes, capaz de reproducir contenidos multimedia o simulaciones que se pueden explorar con el teclado, el mouse, un joystick o una pantalla táctil. Esta forma de RV carece por completo de sensaciones de inmersión para el usuario.

b) En segundo lugar, están los sistema de RV semi-inmersiva, la cual intenta proporcionar a los usuarios una sensación de estar inmersos ligeramente en un entorno virtual, se realiza generalmente mediante diferentes tipos de software y a través de pantallas tridimensionales.

c) El tercer tipo de RV se conoce como de inmersión total, este sistema habitualmente está constituido por un par de pantallas de visualización tridimensional montadas en un casco sobre la cabeza del usuario, lo que le permiten a éste estar completamente aislado del mundo físico exterior. Recientemente, se ha observado un creciente interés en la construcción de las llamadas Cuevas de RV, la cual es una sala en la que las paredes que rodean al usuario producen las imágenes tridimensionales, por lo que ofrece también una sensación de inmersión total; en los ambientes inmersivos es necesario algún tipo de hardware especial para interactuar con el entorno, como son guantes, trajes y sistemas informáticos de alta. La RV totalmente inmersiva es considera por diversas razones la mejor opción para trasmitir información multisensorial, incluyendo entre ellas la capacidad de aislar casi por completo la interferencia que pudiera proveer el mundo exterior y permitir de este modo al usuario enfocarse por completo en la información que le proporciona el entorno virtual.

Actualmente también la Realidad Aumentada es considerada como parte de la RV y consiste en un sistema en el que los usuarios pueden tener acceso a una combinación entre la RV y algunos objetos del mundo real, lo cual es posible mediante la incorporación de objetos gráficos elaborados por computadora dentro de escena del mundo real, a esta tecnología se le conoce también como Realidad Mezclada.

Etapa 6. Continuando con el análisis sistémico en esta etapa de la investigación se exploto el concepto de convergencia tecnológica, encontrándose que un sistema de RV completo a nivel de hardware está integrado por:

1. El equipo generador del entorno virtual: es un sistema de procesamiento y generación de imágenes de alta definición, con gran capacidad para generar gráficos de alto rendimiento. Es considerado el núcleo del sistema de RV y es también el responsable del desempeño, las operaciones y la generación de los escenarios de RV, siendo también el enlace para la conexión y coordinación con los diversos subsistemas del sistema de trabajo y funcionamiento integrando un sistema de RV completo.

2. Los dispositivos de visualización para RV: Hay una variedad muy amplia de sistemas o equipos de visualización, pudiendo ser desde un casco montado sobre la cabeza (HMD), un monitor de gran tamaño, o un sistema de visualización basado en proyectores estereoscópicos. En todos estos sistemas o dispositivos de visualización la proyección tridimensional virtual se utiliza comúnmente como parte de la RV en conjunto con las herramientas y métodos de simulación visual. Permite a los participantes realizar el intercambio de información con el espacio virtual y los escenarios generados.

3. El sistema interactivo de RV: Para Hao-ran \& Jing-bo (citado por Hao y Li, 2010) la interacción en tiempo real es una característica esencial en algunos sistemas de RV, si dicha interacción no se realiza las aplicaciones de RV pierden valor e importancia en su existencia, esta es la diferencia fundamental entre la RV y la animación en tres dimensiones, por lo general es necesario un equipo periférico especial, como son los 
sistemas de retroalimentación de fuerza o táctiles, guantes de datos, sensores de seguimiento o de posición o mouse con varios grados de libertad, entre otros para lograr la interactividad.

4. El Control integrado de RV: Un sistema de RV a gran escala incluye muchos componentes, tales como múltiples proyectores, sistema de sonido y múltiples entradas de vídeo y de conmutación, e incluso control de iluminación y sistemas de cortinas; dichos componentes son necesarios para el control y la gestión adecuada de todos los componentes del sistema, cada parte incluye muchos productos y equipos, estos productos deben conectarse entre sí y trabajar juntos. Sin embargo, a pesar de que el sistema es muy complejo y necesita ser administrado adecuadamente, en la actualidad con el desarrollo tecnológico, esta tarea la realiza el sistema de control integrado logrando que la serie de trabajo necesarios se pueda llevar a cabo con un simple control remoto.

Del análisis anterior fue desarrollada la figura 4, en la que se observa de manera integral la interrelación existente entre diversas tecnologías que convergen en la $\mathrm{RV}$, lo que anteriormente en este trabajo se había definido como convergencia tecnológica, y que fue clave en esta investigación ya que derivado de ella, la RV no sólo se vuelve más accesible para diversas instituciones educativas por sus costos y potencial de procesamiento de información, abriéndose con ello la posibilidad de masificar su utilización (Olawuyi y Mgbole, 2012).

Figura 4: Convergencia tecnológica en el campo de la RV

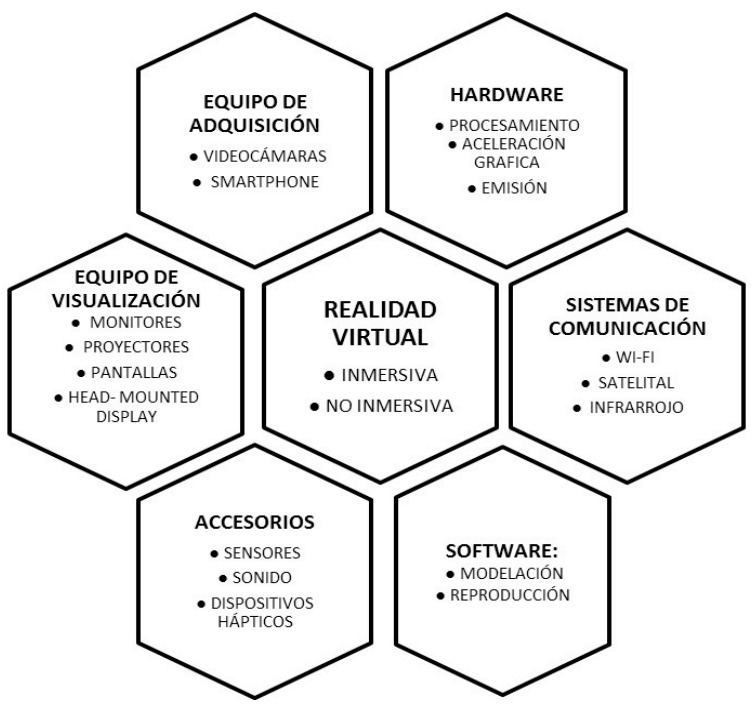

Fuente: Elaboración propia, 2014.

Etapa 7. Para concluir esta investigación se desarrolló el modelo de utilidad que se muestra en la figura 5, el cual opera de la siguiente forma:

1. Determina el tipo de obstáculo didáctico que se tiene en alguna asignatura, es decir si se tiene que definir si se trata de un conceptos abstracto, se genera una situaciones de riesgo, o se requiere un modelo que represente algún equipo o situación de la realidad MRR: Requiere Modelos para Representar la Realidad, RVI: Requiere Visitas Industriales \& RCE: Requiere Conocer Equipo en Campo.

2. Seleccionar que tipo de RV se va a utilizar no inmersiva, semi-inmersiva o inmersiva.

3. Desarrollar los materiales adecuados para el tipo de RV seleccionada, estos materiales van desde una grabación hecha con alguna cámara estereoscópica, hasta modelos tridimensionales desarrollados con algún software especial.

4. Finalmente los estudiantes deben ejecutar el material en el ambiente de RV seleccionado. 
Figura 5: Diagrama esquemático de la aplicación de la RV en asignaturas que presentan algún riesgo eléctrico

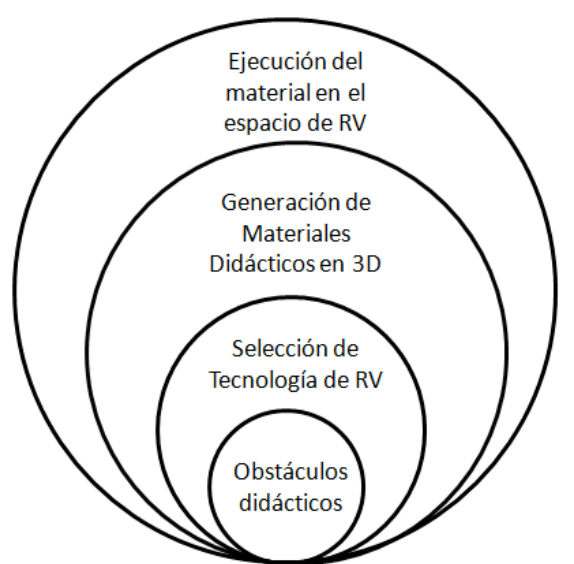

Fuente: Elaboración propia, 2014.

\section{Resultados}

Del análisis sistémico realizado se observó que es posible implementar la tecnología de RV dentro del proceso de enseñanza aprendizaje de los estudiantes de ingeniería eléctrica, para el caso de visitas a subestaciones eléctricas.

Un amplio espectro de los estilos de aprendizaje de los estudiantes es cubierto por las propiedades emergentes que tiene la RV, lo que hace factible su implementación como estrategia didáctica dentro de los centros educativos.

\section{Conclusiones}

Derivado de este estudio se puede concluir que desde el punto de vista teórico la RV es una alternativa tecnológica factible de ser utilizada dentro del procesos formativo de los estudiantes de ingeniería eléctrica, ya que dicha tecnología pueden resultar una estrategia útil para trasmitir información a los estudiantes de ingeniería eléctrica, en aquellas situaciones donde existen condiciones que podrían poner en riesgo la vida. Pero es necesario generar mayor información que se relacione de manera específica con el uso de la RV en el campo de la educación de manera general y de manera específica con el tema de formación de ingenieros. 


\section{REFERENCIAS}

Abulrub, A. G., Attridge, A. \& Williams, M. A. (2011). Virtual Reality in Engineering Education: The Future of Creative Learning. International Journal of Emerging Technologies in Learning (iJET), 6(4), pp. 4-11. Recuperado el 1 de enero de 2013 de: <http://onlinejournals.org/i-jet/article/view/1766/2019>.

Academia de Ingeniería de México. (2010). Prospectiva de la Ingeniería en México y en el Mundo. Consejo Nacional de Ciencia y Tecnología. México.

Ai-Lim, E. \& Wai, K. (2008). A review of using virtual reality for learning. In Abdennour El Rhabili (Ed.), Lecture Notes In Computer Science (pp. 231-241. Berlin: Heidelberg.

Ariyakul, Y., Aizawa, T. \& Nakamoto, T. (2013). Visual-olfactory presentation system using a miniaturized olfactory display based on SAW streaming and electro-osmotic pumps. Virtual Reality (VR), 18(20), pp.155,156.

Bohorquez, H. J., et al. (2009). La Concepción de la Simetría en Estudiantes como un Obstáculo Epistemológico para el Aprendizaje de la Geometría. EDUCERE, 13(45), pp. 477-489.

Bosch, H., et al. (2011). Nuevo Paradigma Pedagógico para Enseñanza de Ciencias y Matemática. Avances en Ciencias e Ingeniería, 2(3), pp. 131-140.

Brudniy, A., Demilhanova, A. (2012). The Virtual Reality in a Context of the "Mirror Stage". International Journal of Advances in Psychology, 1, pp. 6-9.

Camarena, P. (2009). La Matemática en el Contexto de las Ciencias. Innovación Educativa, 9(46), pp. $15-25$.

Chadwick, C. (1992). Tecnología Educacional para el Docente. Paidos Iberica. Barcelona: Editorial Paidós.

Fällman, D., Backman, A. \& Holmlund, K. (1999). VR in Education: An Introduction to Multisensory Constructivist Learning Environments. Universitets pedagogisk konferens, Umeå universitet, pp. 18-19.

Hao X. \& Li M. (2010). Application of Virtual Reality Technology on Teaching of Basic Computer. 3rd International Conference on Computer and Electrical Engineering (ICCEE 2010) IPCSIT, Singapore.

Kalorama Healthcare reports. (2011) Virtual Reality (VR) in Healthcare in the US (Markets for Surgery, Visualization, Rehabilitation and Training). Ed. 2. Kalorama Info 2011.

Leng, J. (2001). Scientific Examples of Virtual Reality and Visualization Applications. Manchester: Research Centre for Computational Science

Maynard N. et al. (2012, julio). Bringing Industry into the Classroom: Virtual Learning Environments for a New Generation. Proceedings of the 8th International CDIO Conference, Brisbane.

Nadan, T., et al. (2011). Is Virtual Reality a Memorable Experience in an Educational Context? International Journal of Emerging Technologies in Learning (iJET), 6(1), pp. 53-57. Recuperado el 10 de enero de $2013<\mathrm{http}$ ://online-journals.org/i-jet/article/view/1433/1666>.

Olawuyi, J.O. y Mgbole, F. (2012). Technological Convergence. Science Journal of Physics, Article ID sjp-221.

Papadakis, S. (2007). Technological convergence: Opportunities and Challenges. Ensayos de la Unión Internacional de Telecomunicaciones, recuperado el 15 de marzo 2013 de: $<$ http://www.itu.int/osg/spu/youngminds/2007/essays/PapadakisSteliosYM2007.pdf>

RTO/NATO. (2003). Virtual Reality: State of Military Research and Applications in Member Countries. RTO Technical Report 18. France.

Sampaio, A. Z. (2000). Virtual Reality Technology Applied in Teaching and Research in Civil Engineering. Education Journal of Information Technology and Application in Education, 1(4), pp. 153-163.

Sulbaran, T., Baker, N.C., (2000), Enhancing engineering education through distributed virtual reality. Frontiers in Education Conference Proceedings. 
Talaba, D. \& Amditis, A. (2008). Tools and Methods Based on Virtual Reality. Product Engineering. The Netherlands: Springer.

Ulloa, G. (2008). ¿Qué pasa con la ingeniería en Colombia?. Eduteka, consultada: Recuperado el 20 de septiembre del 2013 de http://www.eduteka.org/IngenieriaColombia.php

Yamakawa, P. et al. (2010). Modelo Tecnológico de Integración de Servicios para la MyPE Peruana. Lima: Universidad ESAN.

\section{SOBRE LOS AUTORES}

Jesús Alberto Flores Cruz: Estudiante de doctorado, Doctorado en Ingeniería de Sistemas, Instituto Politécnico Nacional (IPN) Ingeniero Electricista y Maestro en Ciencias en Ingeniería de Sistemas, actualmente realizo estudios de doctorado desarrollando la tesis titulada: "Espacio Virtual de Aprendizaje basado en un Sistema de Realidad Virtual Inmersivo para la Formación de Ingenieros Electricistas", desde hace nueve años formó parte del claustro académico de la Escuela Superior de Ingeniería Mecánica y Eléctrica del Instituto Politécnico Nacional, donde me desempeño como profesor Titular "C", dentro del departamento académico de ingeniería eléctrica. He desempeñado diversos puestos dentro de la administración pública, como son subdirector académico, subdirector de relaciones industriales y jefe del departamento de servicios tecnológicos de la Universidad Tecnológica de Nezahualcóyotl, además de ser presidente de la Asociación Mexicana de la Informática para la Ingeniería.

Patricia Camarena Gallardo: Tiene estudios de Licenciatura en Física y Matemáticas de la Escuela Superior de Física y Matemáticas del Instituto Politécnico Nacional. Posee Maestría y Doctorado en Ciencias con especialidad en Matemática Educativa del CINVESTAV-IPN. Está adscrita al Departamento de Ingeniería en Comunicaciones y Electrónica de la Escuela Superior de Ingeniería Mecánica y Eléctrica del Instituto Politécnico Nacional desde 1975 a la fecha. Ha impartido clases de matemáticas en diversas escuelas de ingeniería del IPN, tanto en licenciatura como en posgrado. Ha dirigido tesis en el área de ingeniería en licenciatura y en posgrado. Ha participado en el diseño de los programas de estudio de casi todas las carreras de ingeniería en el IPN. Sus líneas de investigación son: Educación, Educación matemática, Matemáticas aplicadas a la Ingeniería y Educación en línea. Ha dirigido proyectos de investigación relacionados con la ingeniería como: "Desarrollo de emprendedores politécnicos en la industria de comunicaciones y electrónica", "Impacto de la física del estado sólido en la formación de los ingenieros en electrónica y ramas afines", "Modelo matemático de atenuación por lluvia en banda Ka", dentro de los proyectos de la NASA y México, "Modelos matemáticos como etapas de la matemática en el contexto de la ingeniería", "Las competencias y calidad de la ingeniería y las ciencias básicas", entre muchos otros. Tiene más de 130 publicaciones de artículos en revistas y eventos académicos. Es autora de 15 libros de difusión y 15 de investigación.

Elvira Avalos Villarreal: Profesora Investigadora en la Escuela Superior de Ingeniería Mecánica y Eléctrica (ESIME), Sede Zacatenco, del Instituto Politécnico Nacional (IPN), México. Ingeniera Químico por la Universidad Autónoma de México (UNAM), Maestría en Ciencias con Especialidad en Ingeniería Industrial por la Escuela Superior de Ingeniería Mecánica y Eléctrica (ESIME), del Instituto Politécnico Nacional (IPN), México. Doctorado en Ciencias de Sistemas por la City University, Inglaterra. Sus áreas de interés académico y profesional son: Evaluación de Proyectos, Administración de Proyectos, Planeación Estratégica y Sistemas de Información. 